(อ) OPEN ACCESS

\begin{abstract}
- Additional material is published online only. To view please visit the journal online (http://dx.doi.org/10.1136/ jnnp-2020-324478).

'Department of Neurosurgery, Baylor College of Medicine, Houston, Texas, USA

${ }^{2}$ Department of Neurological Surgery, Columbia University Medical Center, New York, New York, USA

${ }^{3}$ Menninger Department of Psychiatry and Behavioral Sciences, Baylor College of Medicine, Houston, TX, USA ${ }^{4}$ Department of Psychiatry, Columbia University Medical Center, New York, New York, USA
\end{abstract}

\section{Correspondence to}

Dr Sameer A. Sheth, Department of Neurosurgery, Baylor College of Medicine, Houston, Texas, USA; Sameer.Sheth@bcm.edu

KRB and YJP contributed equally.

KRB and YJP are joint first authors.

Received 15 July 2020 Revised 24 February 2021 Accepted 24 March 2021 Published Online First 27 April 2021

Check for updates

(C) Author(s) (or their employer(s)) 2021. Re-use permitted under CC BY-NC. No commercial re-use. See rights and permissions. Published by BMJ.

To cite: Bijanki KR, Pathak YJ, Najera RA, et al. J Neurol Neurosurg Psychiatry

2021:92:776-786.

\title{
Defining functional brain networks underlying obsessive-compulsive disorder (OCD) using treatment-induced neuroimaging changes: a systematic review of the literature
}

\author{
Kelly R. Bijanki (1) ,' Yagna J. Pathak, ${ }^{2}$ Ricardo A. Najera, ${ }^{1}$ Eric A. Storch, ${ }^{3}$ \\ Wayne K Goodman, ${ }^{3}$ H. Blair Simpson, ${ }^{4}$ Sameer A. Sheth ${ }^{1}$
}

\section{ABSTRACT}

Approximately $2 \%-3 \%$ of the population suffers from obsessive-compulsive disorder (OCD). Several brain regions have been implicated in the pathophysiology of $O C D$, but their various contributions remain unclear. We examined changes in structural and functional neuroimaging before and after a variety of therapeutic interventions as an index into identifying the underlying networks involved. We identified 64 studies from 1990 to 2020 comparing pretreatment and post-treatment imaging of patients with $O C D$, including metabolic and perfusion, neurochemical, structural, functional and connectivity-based modalities. Treatment class included pharmacotherapy, cognitive-behavioural therapy/exposure and response prevention, stereotactic lesions, deep brain stimulation and transcranial magnetic stimulation. Changes in several brain regions are consistent and correspond with treatment response despite the heterogeneity in treatments and neuroimaging modalities. Most notable are decreases in metabolism and perfusion of the caudate, anterior cingulate cortex, thalamus and regions of prefrontal cortex (PFC) including the orbitofrontal cortex (OFC), dorsolateral PFC (DLPFC), ventromedial PFC (VMPFC) and ventrolateral PFC (VLPFC). Modulating activity within regions of the cortico-striato-thalamo-cortical system may be a common therapeutic mechanism across treatments. We identify future needs and current knowledge gaps that can be mitigated by implementing integrative methods. Future studies should incorporate a systematic, analytical approach to testing objective correlates of treatment response to better understand neurophysiological mechanisms of dysfunction.

\section{INTRODUCTION}

Obsessive-compulsive disorder (OCD) is a chronic, disabling disease characterised by recurrent, intrusive thoughts (obsessions) and/or repetitive mental or physical actions (compulsions). Typical onset occurs during the childhood or adolescence and often lasts through adulthood; lifetime prevalence is estimated at $2.3 \%$ in the American population. ${ }^{1}$ Patients often suffer from impaired interpersonal relationships as a result of their symptoms, and unemployment rates are reported as high as $38 \%{ }^{2}$
Aberrant signalling in cortico-striato-thalamocortical (CSTC) circuits is considered to be an important pathological mechanism underlying OCD. ${ }^{[\mathrm{S} 1-\mathrm{S} 3]}$ These circuits are composed of glutamatergic and GABAergic projections that connect frontocortical and subcortical brain areas. ${ }^{[\mathrm{S4}-\mathrm{S9}]}$ Within the CSTC loop, the direct pathway from striatum to internal pallidum to thalamus and back to cortex exerts a net excitatory effect, and the indirect pathway, which additionally includes the external pallidum and subthalamic nucleus, produces a net inhibitory effect. ${ }^{[54]} \mathrm{A}$ lack of balance in these pathways is hypothesised to lead to a dysregulated thalamo-cortical drive resulting in inappropriate selection of actions and failure to inhibit maladaptive behaviours, both of which are characteristic of the OCD phenotype. ${ }^{[54, S 10-11]}$ CSTC regions most frequently investigated in OCD are regions of prefrontal cortex (PFC) (including orbitofrontal cortex (OFC), dorsolateral PFC (DLPFC), ventromedial PFC (VMPFC) and anterior cingulate cortex (ACC)), striatum and mediodorsal thalamus.

OCD treatment includes pharmacological and cognitive-behavioural therapies (CBT) as well as less-common neuromodulatory strategies, which may be neurosurgical (eg, stereotactic lesions and deep brain stimulation (DBS)) or non-invasive (eg, transcranial magnetic stimulation, TMS). ${ }^{[12-S 13]}$ This review summarises studies that examined the brain before and after treatment to discover changes in the brain that were associated with symptom improvement, with the hypothesis that common neural mediators may be observed regardless of treatment modality. We also identify knowledge gaps and recommendations for future research.

\section{RESULTS}

Our results are organised by treatment and subsequently by imaging modality. Following literature review as summarised (figure 1), in total, we included 64 distinct studies which report changes in neuroimaging markers following treatment and examine correlation with clinical outcome measured by YaleBrown Obsessive Compulsive Scale (Y-BOCS), as the most standardised and validated instrument to quantify OCD symptomatology. Of the studies that reported significant correlations with Y-BOCS, 28 focused on an adult cohort, 5 on a paediatric cohort 


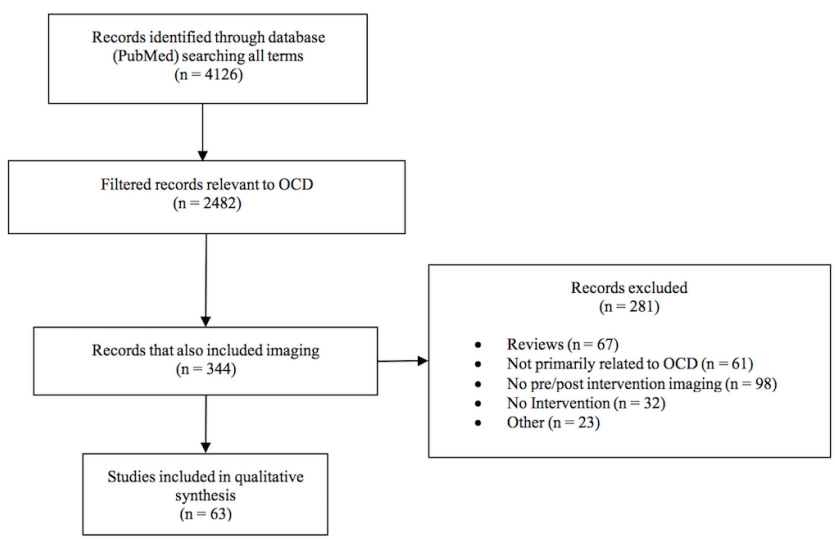

Figure 1 PRISMA flow chart. OCD, obsessive-compulsive disorder; PRISMA, Preferred Reporting Items for Systematic Reviews and MetaAnalyses.

and 1 included patients between the ages of 16-29. Within each section, we first summarise statistically significant results from regions most frequently implicated in the CSTC circuit (OFC, DLPFC, VMPFC, ventrolateral PFC (VLPFC), ACC, caudate and thalamus). All other regions that changed significantly are also reported. Laterality is indicated if included in table 1 . At the end of each section, we summarise the subset of results in which neuroimaging changes correlated significantly with treatment response (i.e., decreased Y-BOCS).

\section{Pharmacotherapy}

Studies evaluated imaging changes after administration of serotonin reuptake inhibitors (SRIs) (fluoxetine ${ }^{3-10}$; fluvoxamine ${ }^{71}$; paroxetine ${ }^{812-17}$ and other SRIs ${ }^{8918-20}$; tricyclic antidepressants (TCAs) (clomipramine) $)^{5} 2122$ and after selective SRI (SSRI) augmentation with the atypical antipsychotic risperidone. ${ }^{23} 24$ Patient populations ranged from treatment-naïve children, ${ }^{6} 131517$ to pharmacotherapy-resistant adults ${ }^{24}$ and treatment duration varied from 12 weeks to 1 year. ${ }^{53}$

\section{Metabolic and perfusion studies (fluoro-deoxyglucose positron} emission tomography, single photon emission CT)

- Caudate: Two studies found a decrease in right caudate metabolism that correlated with a decrease in Y-BOCS after SRI pharmacotherapy ( $\mathrm{n}=9$ adults), ${ }^{3}$ ( $\mathrm{n}=20$ adults). ${ }^{14}$ Two studies reported a decrease in caudate metabolism, one on the right ( $\mathrm{n}=20$ adults) ${ }^{16}$ and one on the left ( $\mathrm{n}=8$ adults), ${ }^{21}$ that did not correlate with symptom reduction after SRI or TCA administration. One study found increased metabolism in the right caudate that correlated with a decrease in Y-BOCS after administering paroxetine ( $\mathrm{n}=7$ adults). ${ }^{12}$ Two other studies showed no significant change in caudate metabolism ( $\mathrm{n}=13$ adults), ${ }^{5}$ ( $\mathrm{n}=11$ adults). ${ }^{7}$ A single photon emission CT (SPECT) study reported a decrease in regional cerebral blood flow (rCBF) in the left caudate after fluvoxamine; however, this finding did not correlate with Y-BOCS reduction ( $\mathrm{n}=15$ adults). ${ }^{11}$ Another SPECT study noted no such change in caudate perfusion after administration of clomipramine ( $n=8$ adults). ${ }^{22}$

- OFC: One study found a decrease in bilateral OFC metabolism that correlated with a decrease in Y-BOCS after SRI or TCA pharmacotherapy ( $\mathrm{n}=13$ adults). ${ }^{5}$ Several other studies found a similar decrease in either right $(\mathrm{n}=20$ adults $),{ }^{16}$ $\left(\mathrm{n}=8\right.$ adults $^{21}$ or bilateral $(\mathrm{n}=10 \text { adults })^{8}$ OFC metabolism after SRI or TCA intervention; however, these did not correlate with symptom reduction. Three studies reported no significant change in OFC metabolism with SRI or TCA treatment $\left(\mathrm{n}=9\right.$ adults), ${ }^{3}$ ( $\mathrm{n}=11$ adults), ${ }^{7}$ ( $\mathrm{n}=7$ adults). ${ }^{12}$ SPECT studies found decrease in OFC perfusion bilaterally that did not correlate with reduction in Y-BOCS $(n=8$ adults). ${ }^{22}$ Others noted no change in OFC metabolism after fluvoxamine $\left(n=15\right.$ adults) ${ }^{11}$ or fluoxetine ( $n=6$ adults). ${ }^{4}$

- PFC/ACC: One fluoro-deoxyglucose positron emission tomography (FDG-PET) study noted decreased metabolism in the bilateral ACC that correlated with decreased Y-BOCS $\left(\mathrm{n}=11\right.$ adults). ${ }^{7}$ Another study reported decreased metabolism in the right ACC that did not, however, correlate with symptom improvement ( $n=9$ adults). ${ }^{3}$ Several others noted no significant change in ACC metabolism corresponding with SRI or TCA outcomes ( $\mathrm{n}=13$ adults), ${ }^{5}\left(\mathrm{n}=20\right.$ adults),${ }^{14}$ ( $\mathrm{n}=20$ adults), ${ }^{16}$ ( $\mathrm{n}=8$ adults). ${ }^{21}$

- Thalamus: One study noted a decreased metabolism in the left thalamus after fluoxetine administration; however, this finding did not correlate with Y-BOCS reduction $\left(n=9\right.$ adults). ${ }^{3}$ Other studies that used SRI or TCA pharmacotherapy found no corresponding significant metabolic change in the thalamus ( $n=20$ adults), ${ }^{16}$ ( $n=8$ adults), ${ }^{21}$ $\left(n=11\right.$ adults). ${ }^{7}$ Results from perfusion studies in the thalamus varied depending on treatment class. Two studies showed rCBF decreases in the right thalamus after SRIs $\left(\mathrm{n}=11\right.$ adults), ${ }^{18}$ but only one reported a significant correlation with Y-BOCS improvement ( $\mathrm{n}=15$ adults). ${ }^{11}$

- Other Regions: Decreased metabolism in bilateral middle and posterior cingulate cortices correlated with Y-BOCS reduction $\left(n=11\right.$ adults). ${ }^{7}$ Another study found both a decrease in metabolism in the right putamen, bilateral cerebella and right hippocampus and an increase in metabolism in the right lateral postcentral gyrus, posterior and superior parietal lobes, and medial and superior occipital gyri after SRI treatment $\left(\mathrm{n}=10\right.$ adults). ${ }^{8}$ Conversely, others noted an increase in metabolism in the putamen bilaterally $(n=8$ adults). ${ }^{21}$ These findings from these studies were not correlated with Y-BOCS reduction.

In contrast to the FDG-PET study results after SRI or TCA treatment, risperidone augmentation (ie, SSRI+ risperidone) resulted in increased metabolism across all CSTC ROIs, including the caudate, OFC, PFC, and ACC, but a decrease in the thalamus; none of these results correlated with symptom response ( $n=16$ adults). ${ }^{24}$ A SPECT study reported increased $\mathrm{rCBF}$ in the bilateral thalami that was correlated with Y-BOCS reduction after augmentation with risperidone; however, no significant change in caudate perfusion was noted ( $n=12$ adults). ${ }^{23}$

Neurochemical studies ( ${ }^{1} \mathrm{H}-\mathrm{MRS}, \alpha-(11 \mathrm{C})$ methyl-L-tryptophan-PET)

- Caudate: After paroxetine therapy, paediatric patients showed a decrease in glutamate concentration (Glx) in the left caudate that correlated with CY-BOCS $(n=11$ children). ${ }^{15}$

- PFC/ACC: After citalopram treatment, N-acetylaspartate (NAA) concentrations in the ACC and PFC increased, suggesting improved neuronal and axonal integrity; however, these did not correlate with symptom response ( $\mathrm{n}=13$ adults). ${ }^{20}$

- Other Regions: One study used PET imaging with an $\alpha-(11 \mathrm{C})$ methyl-L-tryptophan tracer to examine the changes in brain regional serotonin synthesis following treatment with sertraline ( $\mathrm{n}=8$ adults) compared with CBT. After sertraline 


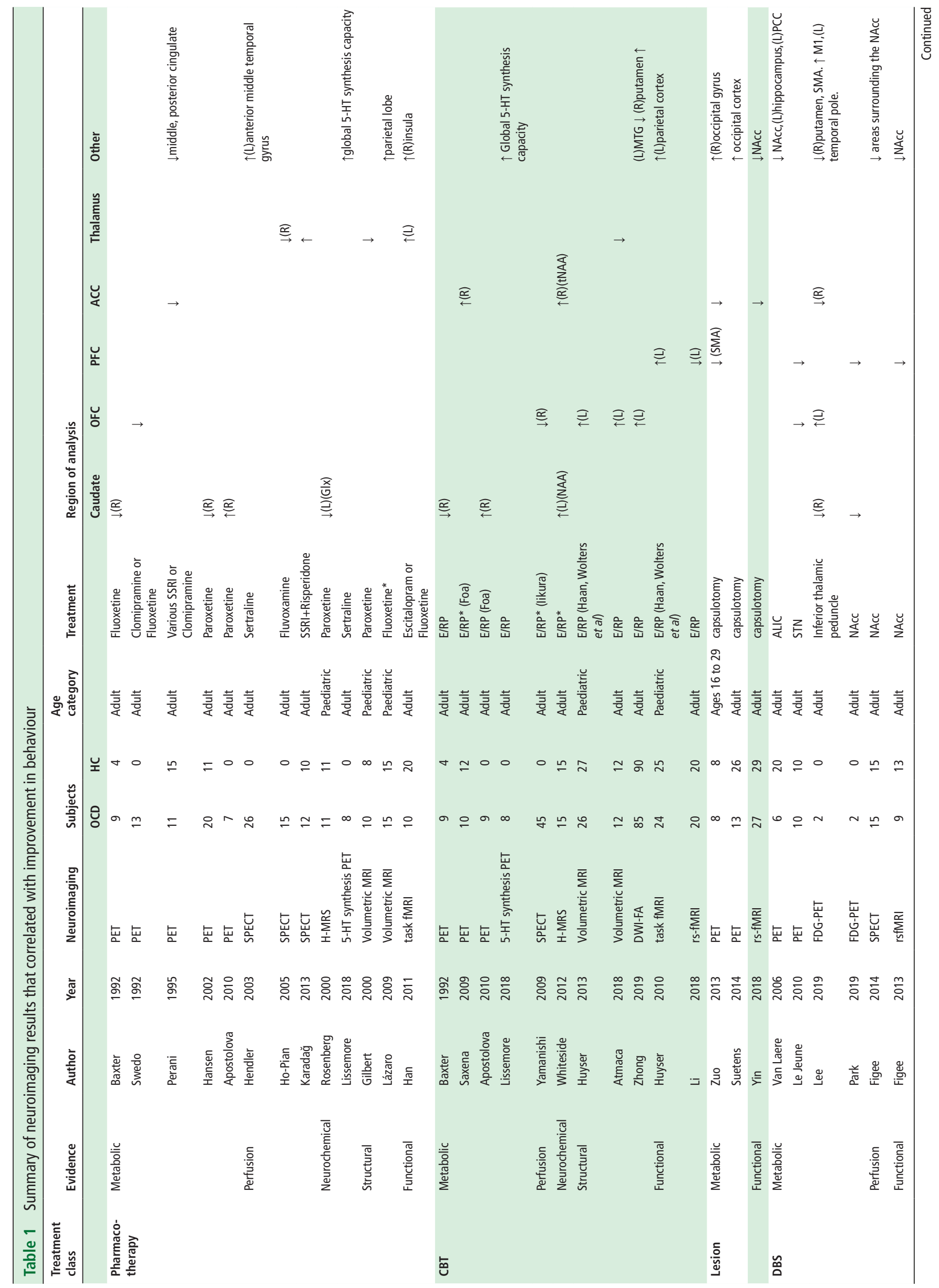


treatment, in treatment responders and partial responders, serotonin synthesis capacity increased brain-wide. ${ }^{25}$ This change was correlated with Y-BOCS reduction.

Structural studies (volumetric MRI and DTI)

- Thalamus: One paroxetine study reported a decrease in bilateral thalamic volumes that correlated with symptom response ( $\mathrm{n}=10$ children). ${ }^{13}$

- Other Regions: Another paroxetine study noted decreased volume in the left amygdala that correlated with SRI dosage but not with change in CY-BOCS ( $\mathrm{n}=11$ children). ${ }^{17}$ Two other studies investigated the effects of fluoxetine on grey matter volumes. Paediatric OCD subjects showed increased bilateral parietal lobe volumes that correlated with reduction in CY-BOCS ( $\mathrm{n}=15$ children). ${ }^{6}$ The adults showed increased volumes of the left putamen after medication; however, this was not correlated with Y-BOCS reduction ( $\mathrm{n}=13$ adults). ${ }^{10}$ A diffusion-weighted MRI study demonstrated that fractional anisotropy (FA) in the corpus callosum, internal capsule, and white-matter in the area superolateral to the right caudate in drug-naïve patients decreased after citalopram ( $\mathrm{n}=13$ adults and matched controls). ${ }^{26}$ This finding was not correlated with treatment response.

Functional MRI studies (task fMRI and resting-state fMRI (rs-fMRI)) One study evaluated functional changes (BOLD signal) after escitalopram ( $\mathrm{n}=9$ adults) or fluoxetine ( $\mathrm{n}=1$ adults) therapy using a task-switching paradigm during functional MRI (fMRI) $\left(\mathrm{n}=10\right.$ adults). ${ }^{9}$

- CSTC: OFC, rostral ACC and VLPFC showed increased activation, however, these increases were not correlated with treatment response.

- Other regions: Increased activation in the left thalamus and the right insula were correlated with reduction in Y-BOCS, but increased activation in hippocampus was not correlated with symptom change.

Summary of neuroimaging changes with pharmacotherapy that correlated significantly with symptom change (ie, significant decrease in Y-BOCS)

- CSTC:

- Caudate: OCD symptom reduction correlated with decreased metabolic activity. ${ }^{314}{ }^{15}$ but see ${ }^{12}$ (figure 2A, table 1)

- OFC and ACC: therapeutic effect correlated with decreased metabolism bilaterally. ${ }^{57}$

- Thalamus: treatment response correlated with decreased perfusion, ${ }^{11}$ decreased volume ${ }^{13}$ and increased BOLD signal during a task-switching paradigm. ${ }^{9}$ However, risperidone augmentation to SSRI produced increased perfusion. ${ }^{23}$

- Outside CSTC:

- mid-cingulate cortex (MCC), posterior cingulate cortex (PCC): decreased metabolic activity. ${ }^{7}$

- Middle temporal gyrus (MTG): increased perfusion. ${ }^{19}$

- Insula: increased BOLD signal in insula. ${ }^{9}$

\section{Cognitive-behavioural therapy}

CBT consisting of exposure and response prevention (ERP) is an evidence-based treatment for OCD, in both adults ${ }^{\left[{ }^{[17]}\right.}$ and children. ${ }^{[18]}$ ERP asks patients to expose themselves to their individual symptom triggers while refraining from ritualising. ERP studies included in this review varied in frequency and duration 

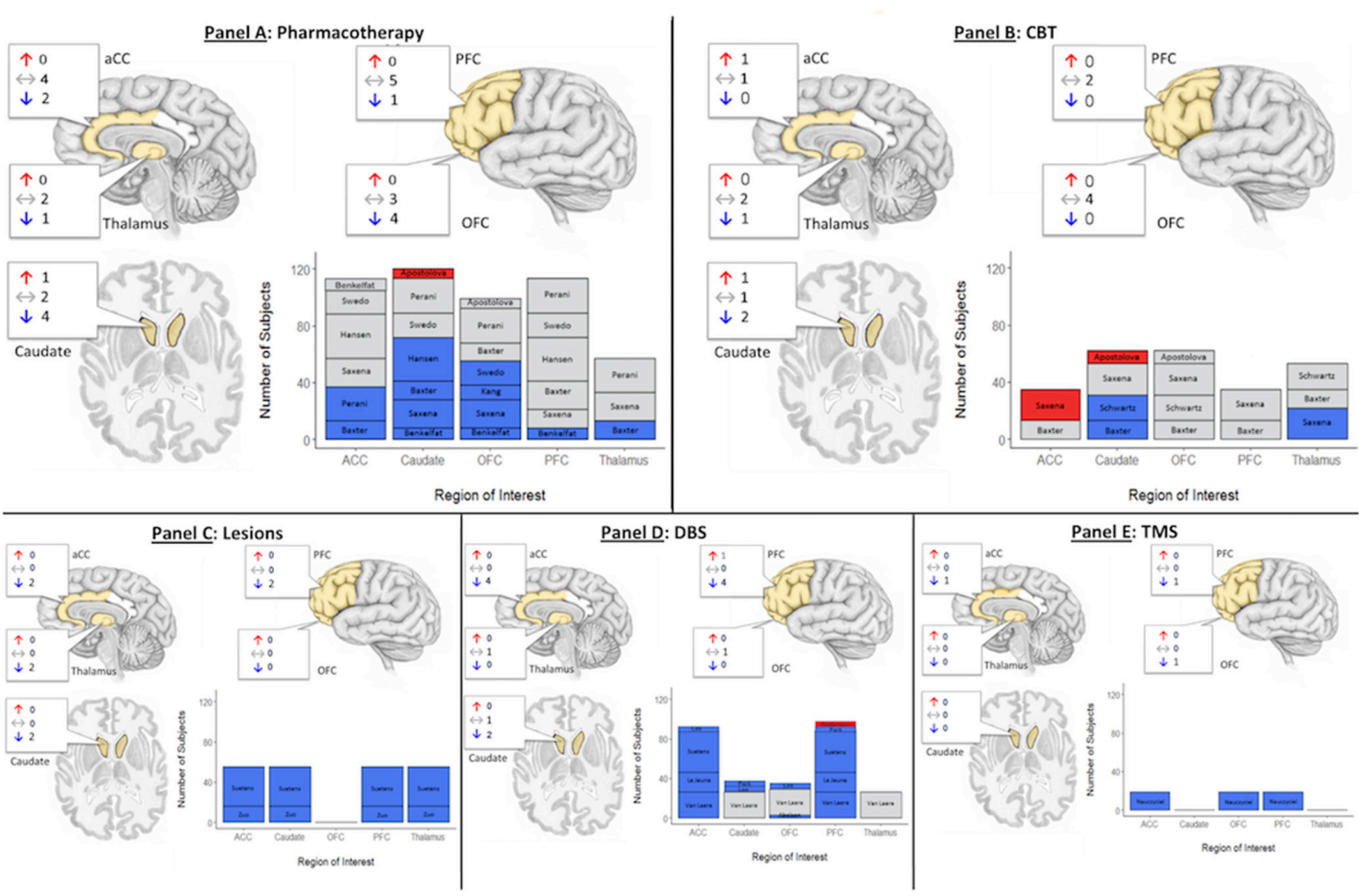

Figure 2 Synthesis of statistically significant changes in brain glucose metabolism correlated with symptom improvement quantified by Yale-Brown Obsessive Compulsive Scale figure 2. Summary of metabolic changes in regions of interest (ACC, caudate, OFC, PFC and thalamus) measured with PET following interventions with (A) pharmacotherapy, (B) CBT, (C) lesions, (D) DBS and (E) TMS. Anatomy call-outs indicate the number of studies reporting increases (red), no change on purposive measurement (grey arrows and boxes) or decreases (blue). Same colours used in bar chart scaled on number of subjects enrolled per trial, labelled by study first author surname. ACC, anterior cingulate cortex; CBT, cognitive-behavioural therapy; DBS, deep brain stimulation; OFC, orbitofrontal cortex; PET, positron emission tomography; PFC, prefrontal cortex.

(ie, 'dosing') of sessions, ranging from intensive strategies (eg, 5 sessions per week for 4 weeks) ${ }^{27-29}$ to gradual (eg, 1-3 sessions per week for 7-20 weeks) treatment courses. ${ }^{312} 30$ Some studies also supplemented CBT with other techniques. Thus, differences reported below may be due to variability in the therapy provided. Patient populations ranged from adults, ${ }^{3} 12282931-36$ to children. ${ }^{30}{ }^{37-41}$ Literature suggests that structural deficits in OCD patients are correlated with age and may represent the pediatric-to-adult progression of the disorder. ${ }^{[19-S 20]}$ Other variabilities included, maintenance of their pharmacological treatment during CBT, 2829343637 a 2-week medication wash-out ${ }^{331-33}$ and 'medication free'30 39 or 'medication naïve' protocols. ${ }^{38} 41$ Lastly, in terms of comparators, most studies did not include treatment-naïve controls; some studies included a treatment comparator, typically pharmacotherapy, ${ }^{3} 12$ or a healthy control arm (table 1). $32930363942-44$

\section{Metabolic and perfusion studies (FDG-PET, SPECT)}

- Caudate: Two studies noted a decrease in caudate metabolism (bilateral caudate; $n=9$ adults), ${ }^{33}$ one of which was correlated with a decrease in Y-BOCS (right caudate; $n=9$ adults). ${ }^{3}$ Conversely, one study showed an increase in right caudate metabolism that also correlated with Y-BOCS reduction ( $n=9$ adults). ${ }^{12}$ One perfusion study found decreased $\mathrm{rCBF}$ in the right caudate that did not correlate with symptom response ( $\mathrm{n}=22$ adults). ${ }^{35}$

- PFC/ACC: One study showed increased metabolism in right dorsal ACC after CBT correlated with symptom improvement ( $\mathrm{n}=9$ adults). ${ }^{29}$ A second study showed decreased perfusion in the right OFC after CBT that correlated significantly with symptom reduction, while decreases in right VMPFC and left middle frontal gyrus did not $(n=45$ adults). ${ }^{34}$

- Thalamus: Saxena et al also reported decreased thalamic metabolism that did not correlate with Y-BOCS reduction. ${ }^{29}$

Neurochemical studies ( ${ }^{1} \mathrm{H}-\mathrm{MRS}, \alpha-(11 \mathrm{C})$ methyl-L-tryptophan-PET)

- Caudate: One study showed an increase in NAA in the left head of the caudate that was correlated with improvement in Y-BOCS ( $\mathrm{n}=15$ adults). ${ }^{36}$ In paediatric patients, one study found that a decrease in Glx concentration in the right head of the caudate was not correlated with symptom response and no change was observed in the pregenual ACC $(n=11$ children). ${ }^{37}$ A separate study, however, found no change in the caudate in a cohort with similar age and treatment duration ( $n=21$ children). ${ }^{38}$

- PFC/ACC: Another study noted an increase in NAA concentrations in the right pregenual ACC and a decrease in Glx in the left middle ACC after an intensive 4-week treatment; however, these findings did not correlate with improvement in Y-BOCS ( $\mathrm{n}=8$ adults). ${ }^{28}$ A paediatric study ( $\mathrm{n}=24$ children) also noted a decreased in Glx in the pACC after 12-14 weeks of CBT. ${ }^{[\mathrm{S} 21]}$ The authors did not report if the change in Glx was correlated with CY-BOCS improvement, but did note that baseline Glx in vACC was a predictor of post-CBT change in CY-BOCS score.

- Other Regions: In a study using an $\alpha$-(11C)methyl-Ltryptophan tracer to examine changes in regional serotonin synthesis following treatment with sertraline compared with CBT ( $\mathrm{n}=8$ adults), treatment responders 
and partial responders demonstrated increased serotonin synthesis capacity brain-wide. that correlated with Y-BOCS reduction. $^{25}$

\section{Structural studies (volumetric MRI and DTI)}

- PFC/ACC: Using voxel-based morphometry (VBM), a paediatric study of medication-free OCD patients $(n=26$ children and 27 controls) showed increased left OFC grey matter volumes after CBT correlated with greater symptom reduction. Additionally, the authors reported decreased volumes in the right globus pallidus and bilateral external capsules that did not correlate with treatment response. ${ }^{39}$ Another study demonstrated unilateral increases in left OFC that correlated with improved Y-BOCS score. ${ }^{42}$

- Thalamus: That study also demonstrated bilateral decreases in thalamic volumes following CBT that correlated with improvement in Y-BOCS scores ( $n=12$ adults). ${ }^{42}$

- Other regions: A diffusion-weighted MRI study used VBM to quantify differences in FA across the middle frontal gyrus, OFC, cerebellum and MTG. In addition, the group noted reductions of FA in the putamen. Among these, the putamen, MTG and OFC findings were correlated with the Y-BOCS reduction ( $\mathrm{n}=85$ adults). ${ }^{43}$

\section{Functional studies (task fMRI and rs-fMRI)}

- Caudate: A reversal learning study showed increased activation in the caudate after CBT that was not correlated with symptom response ( $\mathrm{n}=10$ adults). ${ }^{27}$

- PFC/ACC: Studies using Stroop, ${ }^{31}$ and symptom provocation. ${ }^{32} 45$ paradigms showed decreased activation in the ACC ( $n=10$ adults) ${ }^{32}(n=31 \text { adults })^{45}$ and $\operatorname{OFC}^{32}(n=11$ adults), ${ }^{31}$ following CBT that did not correlate with Y-BOCS. A resting-state connectivity study demonstrated decreased resting-state functional connectivity (RSFC) between left DLPFC and right precuneus, right superior temporal gyrus and cuneus that correlated with reduction in Y-BOCS $(n=20$ adults). ${ }^{44}$ In contrast, one study, using the Tower of London executive function task, showed increased BOLD signal in the left DLPFC and in the left parietal cortex following CBT that were correlated with symptom response $(n=24$ children). ${ }^{30}$

- Other regions: A RSFC study showed reduced connectivity in the default mode network and the task-positive network in the treatment-naïve OCD cohort ( $n=25$ children) at baseline compared with healthy controls ( $n=23$ children). While this group gathered neuroimaging data before and after CBT, they reported no significant change in fMRI-based connectivity as a function of OCD symptom measurements. ${ }^{41}$

\section{Summary of neuroimaging changes with CBT that correlated} significantly with symptom change

- Decreased metabolism in the caudate (figure 2B, table 1). ${ }^{3}$

- Increased metabolism in dorsal ACC. ${ }^{29}$

- Decreased perfusion in OFC. ${ }^{34}$

- In children, increased volume of OFC, ${ }^{39}$ increased activation of DLPFC and left parietal cortex during an executive function task. ${ }^{30}$

- Increased FA of MFG, OFC, cerebellum and MTG. ${ }^{43}$

\section{Stereotactic lesions}

Stereotactic lesions have been used in patients with severe, intractable OCD since the 1960 s. $^{[\mathrm{S} 22-\mathrm{S} 23]}$ These lesions target nodes (eg, dorsal anterior cingulotomy) ${ }^{[\mathrm{S} 24-\mathrm{S} 25]}$ or white matter connections between nodes (eg, anterior capsulotomy, ${ }^{[\mathrm{S} 26-\mathrm{S} 27]}$ subcaudate tractotomy $)^{[\mathrm{S} 28]}$ in the CSTC circuit. $^{[\mathrm{S} 22]}$ Studies included in this review implemented thermoablative anterior capsulotomy, ${ }^{46} 47$ radiosurgical (Gamma Knife) anterior capsulotomy, ${ }^{48}$ thermoablative cingulotomy ${ }^{49}$ and thermoablative limbic leucotomy. ${ }^{50}$ Patients undergoing lesion procedures have failed pharmacotherapy and CBT. ${ }^{51}$ The severity and intractability of their disease, potential long-term exposure to medications, and frequent comorbid psychiatric conditions, such as major depressive disorder, distinguish them from the patient cohorts in previous categories. ${ }^{52}$

\section{Metabolic and perfusion studies (FDG-PET, SPECT)}

FDG-PET before and after anterior capsulotomy $(\mathrm{n}=8$, 16-29years) demonstrated decreased metabolism in bilateral dorsal ACC, and OFC, as well as increased metabolism in middle and inferior occipital gyri correlated with decreased Y-BOCS. Other regions showed changes not correlated with Y-BOCS: decreased metabolism in bilateral caudate, medial dorsal thalamus, and right cerebellar tonsil, and increased metabolism in precentral and superior temporal gyri. ${ }^{47}$

Another FDG-PET study compared anterior capsulotomy ( $n=13$ adults) with DBS. After capsulotomy, metabolism decreased in the left caudate head and body, bilateral medial frontal cortices, right inferior frontal cortex, left dACC and left medial dorsal thalamus, and bilateral posterior cerebellum. Metabolism increased in right cuneus and bilateral parietal lobes. Only changes in the occipital lobe correlated with decreased Y-BOCS. ${ }^{46}$

SPECT showed rCBF decreased in the right PFC and the left striatum following limbic leucotomy, but changes were not significantly correlated with Y-BOCS change ( $\mathrm{n}=8$ adults). ${ }^{50}$ Another group found $\mathrm{rCBF}$ decreased in paraterminal gyri and increased bilaterally in DLPFC and left lateral temporal lobe following bilateral anterior capsulotomy ( $n=3$ adults). These postoperative changes were interpreted to reflect connectional diaschisis, such that the procedure served to disconnect the DLPFC and lateral temporal lobe from hyperactive paraterminal gyri. Due to the small sample size, the team did not correlate SPECT changes with Y-BOCS change. ${ }^{53}$

\section{Structural studies (volumetric MRI and DTI)}

Volumetric decrease in the caudate body was shown following thermoablative cingulotomy ( $\mathrm{n}=9$ adults) ${ }^{49} \mathrm{VBM}$ analysis found non-significant changes to caudate, thalamus and ACC following radiosurgical capsulotomy, instead showing a significant increase in right inferior frontal gyrus ( $\mathrm{n}=5$ adults). ${ }^{48}$ Changes in both studies were unrelated to symptomatic response.

\section{Functional studies (task fMRI and rs-fMRI)}

Decreased RSFC between the striatum and dorsal ACC correlated with symptom response in patients who underwent capsulotomy ( $\mathrm{n}=27$ adults). ${ }^{54}$

Summary of neuroimaging changes with lesions that significantly correlated with symptom change

- Increase in metabolism in the middle and inferior occipital gyri after capsulotomy (figure 2C, table 1). ${ }^{46} 47$

- Decreased metabolism in dorsal ACC and OFC after capsulotomy. ${ }^{47}$

- Decrease in connectivity between the striatum and dorsal ACC after capsulotomy. ${ }^{54}$ 


\section{Deep brain stimulation}

Like stereotactic lesions, DBS is used to treat patients with severe, refractory OCD by targeting nodes and pathways within the CSTC. ${ }^{\left[{ }^{S 29-S 33]}\right.}$ Unlike lesions, DBS is adjustable and reversible. DBS for OCD typically targets the anterior limb of the internal capsule (ALIC), a large white matter pathway running between the caudate nucleus and the ventral striatum (VS). ${ }^{46-57}$ Targeting has become increasingly refined; groups have defined idealised portions of the ALIC to target based on their connectivity and proximity to CSTC-affiliated structures. Common targets include the ventral capsule/VS (VC/VS) ${ }^{58}$ nucleus accumbens (NAcc) ${ }^{59}$ and bed nucleus of the stria terminalis (BNST), ${ }^{[333]}$ although recent consensus suggests that electrical modulation of the capsular white matter fibres likely drives treatment response. ${ }^{[\mathrm{S} 34]}$ The same caveats regarding severity and intractability noted above for patients undergoing stereotactic lesions apply to patients undergoing DBS.

\section{Metabolic and perfusion studies (FDG-PET, SPECT)}

FDG-PET demonstrates changes in metabolism within CSTC circuits and elsewhere. ALIC stimulation decreased metabolism in the OFC bilaterally ( $\mathrm{n}=4$ adults $)^{56}$; however, this change was not correlated with treatment response. Another study observed ALIC DBS decreased metabolism in the bilateral NAcc, left hippocampus, subgenual ACC, and left PCC that correlated with Y-BOCS reduction. The same study also noted a decrease in metabolic activity in the right insula that did not correlate with improvement in symptoms ( $n=6$ adults). ${ }^{57}$ One study, which included all 6 OCD patients from Van Laere et al, also demonstrated decreased metabolism in the left PCC following DBS ( $n=16$ adults); however, this finding was not correlated with reduction in Y-BOCS. Following stimulation to the region of the $\mathrm{VC} / \mathrm{vs}$, in a subset of these patients, this group further showed that metabolic activity decreased in the subgenual ACC but was not correlated with Y-BOCS score. ${ }^{46}$

FDG-PET showed pretreatment metabolic hyperactivity of the bilateral frontal lobe including ACC compared with controls which was reduced after 4-6 years of therapeutic stimulation to NAcc ( $n=2$ adults). Stimulation was also found to reduce metabolic activity in the caudate nucleus. These changes were correlated with clinical improvement (Y-BOCS). ${ }^{[\mathrm{S} 35]}$

STN stimulation produced decreased metabolism in the VMPFC, ACC and OFC that correlated with Y-BOCS reduction ( $\mathrm{n}=10$ adults). ${ }^{55}$ Another study showed inferior thalamic peduncle stimulation in two patients with OCD produced decreased metabolism in right caudate, putamen, supplementary motor area, and cingulate, as well as increased metabolism in the bilateral primary motor areas, left temporal pole, and left OFC, which were not assessed for direct correlation with Y-BOCS scores. $^{[\mathrm{S} 36]}$

$\mathrm{A}^{15} \mathrm{O}-\mathrm{CO}_{2}$ PET study showed increased $\mathrm{rCBF}$ in right subgenual ACC, right medial OFC, right dorsolateral putamen, and left globus pallidus after 3 months of VC/vs stimulation $(n=6$ adults). ${ }^{58}$ None of these findings correlated with behavioural response (Y-BOCS). Oxygen-15 positron emission tomography $\left({ }^{15} \mathrm{O}\right.$-PET) during acute monopolar VC/VS DBS demonstrated increased perfusion in the $\mathrm{AACC}$ that correlated with a reduction in depressive symptom severity ( $n=6$ adults). There were no significant changes in OCD symptoms. ${ }^{[37]}$ NAcc DBS was evaluated with SPECT, showing decreased D2/3R availability post-DBS correlated with symptom response ( $\mathrm{n}=15$ adults). ${ }^{[\mathrm{S} 37]}$ These findings suggest increased dopamine release causing competition for the radiotracer, diminished D2/3R affinity, and/or structural receptor decrease due to down-regulation.

\section{Functional studies (task fMRI and rs-fMRI)}

An fMRI reward anticipation task during NAcc DBS and showed abnormally low NAcc activity at baseline which normalised to the level of controls after 1 week of stimulation. In a separate resting-state fMRI experiment, the same group found that connectivity (NAcc:left VMPFC) and (NAcc:bilateral VLPFC) decreased after DBS and correlated with symptom reduction (Y-BOCS). They suggested that cortico-striatal disruption may underlie NAcc DBS ( $\mathrm{n}=9$ OCD adults and 13 controls). ${ }^{59}$

\section{Summary of neuroimaging changes with DBS that correlated with} symptom change

- Decreased metabolism in the OFC and VMPFC after STN DBS (figure 2D, table 1). ${ }^{55}$

- Decreased metabolism in CSTC regions following long-term DBS therapy. ${ }^{55} 57$ [S35-S36]

- Reduced rs-fMRI connectivity between the NAcc and bilateral VLPFC, and NAcc and left VMPFC following clinically effective NAcc DBS. ${ }^{59}$

Challenges of studying neuroimaging changes with DBS using MR Unlike in other treatment modalities, the use of MRI in DBS patients is limited by strict safety regulations, due to early findings of adverse effects caused by interactions between magnetic fields and the metal electrodes and coils. ${ }^{[\mathrm{S39}]}$ Despite its technical challenges, some studies have been able to use MRI in DBS. ${ }^{[539]}$ A recent multi-institution study of 102 patients with DBS implants reported no adverse effects related to $1.5 \mathrm{~T}$ or $3 \mathrm{~T}$ MRI. ${ }^{[S 41]}$ However, the potential for artifact-related noise and voxel-effacement remains a challenge. Further investigation is certainly warranted as the use of MRI will be central to understanding the pathophysiology of severe, treatment-refractory OCD.

\section{Transcranial magnetic stimulation}

TMS ${ }^{\left[{ }^{[42]}\right.}$ is a non-invasive technique that induces an electric field on the brain via alternating current in a wire coil held adjacent to the scalp. The induced field produces electrical stimulation in brain regions directly beneath the coil. ${ }^{[\mathrm{S43}-\mathrm{S44}]}$ The parameters of stimulation (coil geometry, frequency, intensity, location) determine the effects. Among repetitive TMS (rTMS) approaches in neuropsychiatry, the most commonly-studied patterns of stimulation are slow $(<1 \mathrm{~Hz})$, fast $(>5 \mathrm{~Hz})$ and theta-burst. ${ }^{[45]}$

\section{Metabolic and Perfusion studies (FDG-PET, SPECT)}

Slow rTMS $(1 \mathrm{~Hz})$ of the right OFC with a butterfly doublecone coil decreased metabolic activity (FDG-PET) in right OFC correlated with Y-BOCS decrease. Right DLPFC, right middle gyrus, right orbital gyrus, left subgenual ACC, and left orbital gyrus decreased in metabolism as well; however, none of these were correlated with Y-BOCS ( $\mathrm{n}=19$ adults). ${ }^{[\mathrm{S46}]}$

\section{Functional Studies (task fMRI, rs-fMRI)}

A recent sham-controlled study evaluated slow rTMS of the right DLPFC region during a provocation task. The active group $(\mathrm{n}=10$ adults) showed no change in BOLD response before vs after stimulation whereas the sham group ( $n=8$ adults) showed a BOLD increase at right inferior frontal gyrus and right putamen; this did not correlate with symptom improvement. ${ }^{[\$ 47]}$ 
Summary of neuroimaging changes with TMS that significantly correlated with symptom change

Low frequency rTMS treatment to right OFC decreased metabolic activity in right $\mathrm{OFC} .^{[\mathrm{S4}]}$ (figure $2 \mathrm{E}$, table 1 )

\section{DISCUSSION}

\section{Summary of main findings}

Through our literature review, we identified several nodes within the CSTC circuit that changed following effective treatments for OCD regardless of treatment modality. We highlight changes that correlated with Y-BOCS decrease, as that correlation suggests a proximal relationship between the neuroimaging finding and symptomatic improvement. Such correlation occurred in thirtyfour of the reviewed studies, as summarised in table 1.

- OFC: decreased activity. ${ }^{5} 81621223132345556$ [S46]

- PFC: decreased metabolism, ${ }^{21} 46475557[\mathrm{S35,546]}$ and decreased perfusion. ${ }^{1850}$

- ACC: decreased activity. 3732464754 57[S36]

- Thalamus: decreased activity. ${ }^{3} 3247$ [S67]

- Caudate: decreased activity. ${ }^{311}$ 14-162133353749[S35-S36]

- But see, ${ }^{9} 12202324$ 27-2936

- NAcc: reduced connectivity with other networks, ${ }^{54} 5759$ [S38]

- Regions outside CSTC exhibited changes:

- Hippocampus: decreased metabolism,

- Posterior Cingulate: decreased metabolism, ${ }^{57}$

- Increased activity in middle/inferior occipital gyri, ${ }^{46} 47$ anterior MTG, ${ }^{19}$ insula, ${ }^{9}$ and parietal cortex. ${ }^{30}$

Characterising OCD pathophysiology in terms of hypoactivity or hyperactivity is a simplification; OCD is a heterogeneous disorder and often, pathophysiology is associated with the particular cluster of symptoms experienced by a particular patient. As noted above, regions such as the caudate show clinically-relevant activity in both directions. Table 1 further details how both hypoactivity and hyperactivity in the CSTC circuit needs remediation for observable clinical improvement. This contradictory evidence further emphasises the need for standardisation and integration of objective measures to parse the mechanisms of OCD pathology. Indeed, perhaps the best framing of the problem would be as a need to 'tune' the CSTC circuit, rather than to increase or decrease activity en masse.

\section{Placement within a conceptual framework}

Neurobiological models of OCD have often focused on dysfunction in mechanisms of cognitive control, ${ }^{[\mathrm{S48}-\mathrm{S} 54]}$ suggesting involvement of ACC, VMPFC and DLPFC, as well as OFC, thalamus and striatum. ${ }^{[\mathrm{S2}, \mathrm{S5} 5]}$ Three sub-domains may contribute to OCD pathophysiology: (1) response selection and inhibition, (2) goal selection and cognitive flexibility and (3) performance monitoring. These processes include recognising relevant stimuli, ignoring distracting influences, inhibiting prepotent responses, selecting optimal responses, monitoring the outcome of actions and adjusting future behaviour based on feedback and evolving goals. ${ }^{[55]}$ Beyond cognitive control, abnormality in reward-seeking in $\mathrm{OCD}^{[\mathrm{S} 38, \mathrm{~S} 56, \mathrm{~S} 57]}$ is being examined dynamically, considering that symptoms may arise from a failure to balance between goal-directed and habit-like behaviour. ${ }^{[\mathrm{S} 58, \mathrm{~S} 59]}$ In addition, abnormal perception and valuation of threat ${ }^{[560-561]}$ may underlie the features of anxiety that frequently occur with OCD. ${ }^{\left[{ }^{662]}\right.}$ For a review of psychological models of OCD, please see. ${ }^{[\mathrm{S} 63]}$

Within the framework of cognitive control, a failure of inhibition-the ability to suppress or delay actions-has been hypothesised to manifest into compulsions (failure to inhibit negative behavioural processes) ${ }^{[55,564]}$ and to a lesser extent it may also contribute to obsessions (failure to inhibit negative cognitive processes $)^{[\mathrm{S65}]}$ in OCD. It is classically thought to explain the repetitive behaviours of OCD, which cannot be effectively suppressed despite patients' desire to stop. Models focused on response selection and inhibition ${ }^{[552]}$ emphasise ACC's role in inhibition, as well as the involvement of DLPFC and OFC, all of which feature prominently in the CTSC circuits.

Cognitive flexibility is another characteristic deficit in OCD, wherein individuals may struggle to switch behaviour patterns (ex: reverse a learnt or habitual behaviour). ${ }^{[558,566]}$ In OCD, the inability to switch behaviours in response to changing stimuli has been associated with hypoactivity in the DLPFC-dorsolateral caudate loop and the rostral putamen, whereas ritualistic, compulsive behaviour is associated with hyperactivity in the OFC-ventromedial caudate loop. ${ }^{[\mathrm{S} 5,10,567-568, \text { S79-S81] }}$

\section{Future directions}

Need for Standardisation

This review reveals the need to standardise future research over several categories.

\section{Treatment delivery}

Interventions are delivered under parameters that determine their clinical and neurobiological effect. Studies vary most commonly in duration of treatments. While dosage is relatively well-defined for pharmacotherapy, different doses have been used in CBT (eg, daily vs weekly treatment), and neuromodulatory 'dose' (eg, frequency, amplitude, duration of therapy for DBS and TMS) is inconsistent across studies. Differences in these variables (stimulation polarity, field volume, tissue properties) likely account for a substantial portion of the discordance in clinical outcomes often reported in neuromodulation studies. ${ }^{[569-570]}$ Therefore, we recommend comprehensive documentation and reporting of therapeutic parameters to allow comparison of the heterogeneous and complex treatment protocols as currently implemented.

\section{Patient selection}

OCD is an especially heterogeneous disorder. Some studies isolate patient groups by symptom type ${ }^{45}$; others exclude comorbid psychiatric disorders. ${ }^{321}$ Most studies cannot restrict themselves to treatment-naïve subjects for practical reasons, so the majority will include patients with treatment history. Furthermore, neurobiological implications of imaging findings children diverge from those of adult patients, including greater interpretational weight surrounding dendritic pruning and neuroplasticity. To address this issue, it is crucial to clearly document symptom types, comorbidities, and treatment history to facilitate the inclusion of these dimensions in future analyses. Lastly, we recommend including a control group for comparison to better identify whether the underlying mechanisms of action resulting in change are due to a reversal in pathology towards normalcy or a compensatory pathway to mitigate existing pathology.

\section{Brain regions}

It is essential to reduce the study variability due to broad and inconsistent anatomical definitions of brain regions. Early studies did not provide coordinates of observed changes. Anatomical definitions tend to span several cytoarchitectonic areas, for example, the OFC was defined in some studies as Brodmann Area (BA) $47,{ }^{48}$ in others as BA $10,{ }^{32} 55$ or as BA $11 .^{2434} 3958$ Further, the term PFC is often used to indicate to medial, lateral, 
dorsal or ventral regions without specification. Hence, a more consistent approach, using appropriately specified BA when possible rather than region names, or coordinates from a standardised system will facilitate accurate comparisons between studies. Additionally, region segmentation is affected by image resolution and quality; when working with structural or functional scans, it is important to consider the trade-offs between scanner resolution, voxel dimensions and scan time to determine which parameters will enable adequate study of the target brain regions without imposing extra burden on subjects.

\section{Gaps in knowledge and future opportunities Connectivity}

Future studies will benefit from thorough investigation of the structure and function CSTC and limbic circuits in OCD. Anatomical and functional connectivity studies using diffusion tensor imaging (DTI) and fMRI, considered alongside histological tracer studies can help build our understanding of the physical structure and dynamical interactions of the network underlying OCD. ${ }^{[571-575]}$ This principle is illustrated by a DBS study recently demonstrating that NAcc stimulation reduced hyperconnectivity as measured by rs-fMRI between frontal and striatal regions and restored normal function..$^{59}$ Connectivity approaches such as this can help answer how and why certain patterns of regional changes occur as a result of these interventions.

\section{Whole brain}

While a majority of the changes correlated with symptom improvement involved regions within the CSTC, some other limbic structures including the mesial temporal region, cingulate and insula were frequently implicated as well. ${ }^{[\mathrm{S2}]}$ Observations of increased metabolism in the left mesial temporal lobe following DBS of the ALIC may be a human correlate of modulations in fear extinction observed in rodents after DBS in the homologous area. ${ }^{57[576]}$ Whole-brain neuroimaging approaches can further our understanding of the complex interplay between CSTC and limbic regions, and how they vary by OCD dimension, severity, treatment history, comorbidities and other factors. Though this review was focused on a comparison of pretreatment ersus posttreatment imaging results, there is value in assessing predictive studies to identify relevant diagnostic features. Predictive markers of response would facilitate the matching of patients with optimal treatments, reducing the major patient and healthcare burden imposed by iterative trials of ultimately unsuccessful therapies. Additionally, we can improve our understanding of the relationship between objective measures and clinically relevant outcomes in real-world scenarios, typically missing from controlled, in-clinic evaluations, by incorporating highresolution neuroimaging with digital tracking of symptoms in future studies.

\section{Electrophysiology}

Extensive imaging and electrophysiological tools are at our disposal today. Each modality has a unique purpose, and corresponding limitations in terms of noise, and spatial and temporal resolution. Combining multiple modalities allows for holistic investigation of OCD pathophysiology. Ideally, we want to understand the activity of involved neural elements at the level of single neurons and population synaptic activity (local field potentials, LFP). Electrophysiological recordings from surgical patients who are implanted with intracranial electrodes across the brain can be leveraged to study activity that is correlated with specific symptom domains. These opportunities exist within the
OCD cohort (pathological network), or in other patient groups (epilepsy, movement disorders). Surgical interventions such as stereoelectroencephalography (sEEG) to monitor seizures in epilepsy patients allows us to access the brain with incredible resolution. We assume, based on psychological evaluations, that a reasonable subset of these patients has intact emotional and cognitive processing and thus, we can leverage such clinical situations to study the dynamics of OCD-related networks in patients without OCD. There is a shifting emphasis towards networkbased treatment approaches in neuropsychiatry, ${ }^{[\mathrm{S77}-\mathrm{S} 78]}$ which is essential for better efficacy in heterogeneous conditions such as OCD. By integrating electrophysiology with high-resolution MRI and DTI, we can better identify nodes that are aberrant and propose a targeted approach to treatment.

\section{CONCLUSION}

Several regions within and outside CSTC circuits have shown consistent patterns of change following effective OCD treatment. Within the CSTC, the caudate, OFC, PFC, ACC and thalamus all demonstrated decreases in metabolism and perfusion after successful treatment with medications, CBT, stereotactic lesions or DBS. These results support the iconic imaging studies of Baxter and Schwartz, ${ }^{33}$ who proposed OCD phenotype may arise from CSTC hyperactivity, and that normalisation of activity occurs with successful therapy. This review finds that several other limbic regions (PCC and MTL) share these features. Future studies must take a systematic approach to identifying neuroimaging correlates of symptomatic response, incorporating structural and functional connectivity to understand network dynamics. Doing so will shed light on the mechanisms of dysfunction that arise within affected circuits and provide valuable insight into improved strategies for targeting therapy.

Please refer to online supplemental file 1 for supplemental references.

\section{Twitter Kelly R. Bijanki @KBijanki}

Contributors $K B, Y P$ and $R N$ reviewed the literature, created the figures and drafted the manuscript. ES, WKG, HBS and SAS revised the draft and figures. KB, YP, RN, ES, WKG, HBS and SAS finalised the manuscript.

Funding This manuscript was supported in part by grants to KB from the National Institutes of Health (K01-MH1 16364 and R21-NS104953) and to SAS by the Dana Foundation and McNair Foundation.

Competing interests $\mathrm{KB}$ reports a patent pending unrelated to the submitted work. ES reports personal fees from Elsevier, personal fees from Wiley, personal fees from Oxford, personal fees from American Psychological Association, personal fees from Jessica Kingsley, personal fees from Springer, personal fees from Lawrence Erlbaum, personal fees from Levo Therapeutics, personal fees from International OCD Foundation, grants from NIH, grants from Texas Higher Education Coordinating Board, grants from Greater Houston Community Foundation, grants from Red Cross, grants from Rebuild Texas, all outside the submitted work. HBS reports royalties from UpToDate and Cambridge University Press, and research support of multisite industry sponsored trial of investigational drug for OCD from Biohaven Pharmaceutical, and a stipend from American Medical Association for role as Associate Editor of JAMA Psychiatry. WKG reports grants from NIH, grants from McNair Foundation, grants and personal fees from Biohaven Pharmaceutical, personal fees from Neurocrine Biosciences, other from Medtronic, all outside the submitted work. SAS reports consulting agreements with Boston Scientific, Neuropace, Abbott, and Zimmer Biomet. The remaining authors report there are no competing interests.

Patient consent for publication Not required.

Provenance and peer review Not commissioned; externally peer reviewed.

Supplemental material This content has been supplied by the author(s). It has not been vetted by BMJ Publishing Group Limited (BMJ) and may not have been peer-reviewed. Any opinions or recommendations discussed are solely those of the author(s) and are not endorsed by BMJ. BMJ disclaims all liability and responsibility arising from any reliance placed on the content. Where the content includes any translated material, BMJ does not warrant the accuracy and reliability of the translations (including but not limited to local regulations, clinical guidelines, 
terminology, drug names and drug dosages), and is not responsible for any error and/or omissions arising from translation and adaptation or otherwise.

Open access This is an open access article distributed in accordance with the Creative Commons Attribution Non Commercial (CC BY-NC 4.0) license, which permits others to distribute, remix, adapt, build upon this work non-commercially, and license their derivative works on different terms, provided the original work is properly cited, appropriate credit is given, any changes made indicated, and the use is non-commercial. See: http://creativecommons.org/licenses/by-nc/4.0/.

\section{ORCID iD}

Kelly R. Bijanki http://orcid.org/0000-0003-1624-8767

\section{REFERENCES}

1 Ruscio AM, Stein DJ, Chiu WT, et al. The epidemiology of obsessive-compulsive disorder in the National comorbidity survey replication. Mol Psychiatry 2010;15:53-63.

2 Mancebo MC, Greenberg B, Grant JE, et al. Correlates of occupational disability in a clinical sample of obsessive-compulsive disorder. Compr Psychiatry 2008;49:43-50.

3 Baxter LR, Schwartz JM, Bergman KS, et al. Caudate glucose metabolic rate changes with both drug and behavior therapy for obsessive-compulsive disorder. Arch Gen Psychiatry 1992;49:681-9.

4 Hoehn-Saric R, Pearlson GD, Harris GJ, et al. Effects of fluoxetine on regional cerebral blood flow in obsessive-compulsive patients. Am J Psychiatry 1991;148:1243-5.

5 Swedo SE, Pietrini P, Leonard HL, et al. Cerebral glucose metabolism in childhoodonset obsessive-compulsive disorder. Revisualization during pharmacotherapy. Arch Gen Psychiatry 1992;49:690-4.

6 Lázaro L, Bargalló N, Castro-Fornieles J, et al. Brain changes in children and adolescents with obsessive-compulsive disorder before and after treatment: a voxelbased morphometric MRI study. Psychiatry Res 2009;172:140-6.

7 Perani D, Colombo C, Bressi S, et al. [18F]FDG PET study in obsessive-compulsive disorder. A clinical/metabolic correlation study after treatment. Br J Psychiatry 1995; 166:244-50

8 Kang D-H, Kwon JS, Kim J-J, et al. Brain glucose metabolic changes associated with neuropsychological improvements after 4 months of treatment in patients with obsessive-compulsive disorder. Acta Psychiatr Scand 2003;107:291-7.

9 Han JY, Kang D-H, Gu B-M, et al. Altered brain activation in ventral frontal-striatal regions following a 16-week pharmacotherapy in unmedicated obsessive-compulsive disorder. J Korean Med Sci 2011;26:665-74.

10 Hoexter MQ, de Souza Duran FL, D'Alcante CC, et al. Gray matter volumes in obsessive-compulsive disorder before and after fluoxetine or cognitive-behavior therapy: a randomized clinical trial. Neuropsychopharmacology 2012;37:734-45.

11 Ho Pian KL, van Megen HJGM, Ramsey NF, et al. Decreased thalamic blood flow in obsessive-compulsive disorder patients responding to fluvoxamine. Psychiatry Res 2005;138:89-97.

12 Apostolova I, Block S, Buchert R, et al. Effects of behavioral therapy or pharmacotherapy on brain glucose metabolism in subjects with obsessive-compulsive disorder as assessed by brain FDG PET. Psychiatry Res 2010;184:105-16.

13 Gilbert AR, Moore GJ, Keshavan MS, et al. Decrease in thalamic volumes of pediatric patients with obsessive-compulsive disorder who are taking paroxetine. Arch Gen Psychiatry 2000;57:449-56.

14 Hansen ES, Hasselbalch S, Law I, et al. The caudate nucleus in obsessive-compulsive disorder. reduced metabolism following treatment with paroxetine: a PET study. Int J Neuropsychopharmacol 2002:5:1-10.

15 Rosenberg DR, MacMaster FP, Keshavan MS, et al. Decrease in caudate glutamatergic concentrations in pediatric obsessive-compulsive disorder patients taking paroxetine. J Am Acad Child Adolesc Psychiatry 2000;39:1096-103.

16 Saxena S, Brody AL, Maidment KM, et al. Localized orbitofrontal and subcortical metabolic changes and predictors of response to paroxetine treatment in obsessivecompulsive disorder. Neuropsychopharmacology 1999;21:683-93.

17 Szeszko PR, MacMillan S, McMeniman M, et al. Amygdala volume reductions in pediatric patients with obsessive-compulsive disorder treated with paroxetine: preliminary findings. Neuropsychopharmacology 2004;29:826-32.

18 Carey PD, Warwick J, Niehaus DJH, et al. Single photon emission computed tomography (SPECT) of anxiety disorders before and after treatment with citalopram. BMC Psychiatry 2004;4:30

19 Hendler T, Goshen E, Tzila Zwas S, et al. Brain reactivity to specific symptom provocation indicates prospective therapeutic outcome in OCD. Psychiatry Res 2003; 124:87-103.

20 Jang JH, Kwon JS, Jang DP, et al. A proton MRSI study of brain N-acetylaspartate level after 12 weeks of citalopram treatment in drug-naive patients with obsessivecompulsive disorder. Am J Psychiatry 2006;163:1202-7.

21 Benkelfat C, Nordahl TE, Semple WE, et al. Local cerebral glucose metabolic rates in obsessive-compulsive disorder. patients treated with clomipramine. Arch Gen Psychiatry 1990;47:840-8.

22 Rubin RT, Ananth J, Villanueva-Meyer J, et al. Regional 133xenon cerebral blood flow and cerebral 99mTc-HMPAO uptake in patients with obsessive-compulsive disorder before and during treatment. Biol Psychiatry 1995;38:429-37.
23 Karadağ F, Kalkan Oğuzhanoğlu N, Yüksel D, et al. The comparison of pre- and posttreatment (99m)TC HMPAO brain SPECT images in patients with obsessive-compulsive disorder. Psychiatry Res 2013;213:169-77.

24 Buchsbaum MS, Hollander E, Pallanti S, et al. Positron emission tomography imaging of risperidone augmentation in serotonin reuptake inhibitor-refractory patients. Neuropsychobiology 2006;53:157-68.

25 Lissemore Jl, Sookman D, Gravel P, et al. Brain serotonin synthesis capacity in obsessive-compulsive disorder: effects of cognitive behavioral therapy and sertraline. Trans/ Psychiatry 2018:8:82.

26 Yoo SY, Jang JH, Shin Y-W, et al. White matter abnormalities in drug-naïve patients with obsessive-compulsive disorder: a diffusion tensor study before and after citalopram treatment. Acta Psychiatr Scand 2007;116:211-9.

27 Freyer T, Klöppel $\mathrm{S}$, Tüscher $\mathrm{O}$, et al. Frontostriatal activation in patients with obsessive-compulsive disorder before and after cognitive behavioral therapy. Psychol Med 2011;41:207-16.

$28 \mathrm{O}^{\prime} \mathrm{Neill} \mathrm{J}$, Gorbis E, Feusner JD, et al. Effects of intensive cognitive-behavioral therapy on cingulate neurochemistry in obsessive-compulsive disorder.J Psychiatr Res 2013;47:494-504

29 Saxena S, Gorbis E, O'Neill J, et al. Rapid effects of brief intensive cognitive-behavioral therapy on brain glucose metabolism in obsessive-compulsive disorder. Mol Psychiatry 2009;14:197-205.

30 Huyser C, Veltman DJ, Wolters LH, et al. Functional magnetic resonance imaging during planning before and after cognitive-behavioral therapy in pediatric obsessivecompulsive disorder. J Am Acad Child Adolesc Psychiatry 2010;49:1238-48. 1248 e1-5.

31 Nabeyama M, Nakagawa A, Yoshiura T, et al. Functional MRI study of brain activation alterations in patients with obsessive-compulsive disorder after symptom improvement. Psychiatry Res 2008;163:236-47.

32 Nakao T, Nakagawa A, Yoshiura T, et al. Brain activation of patients with obsessivecompulsive disorder during neuropsychological and symptom provocation tasks before and after symptom improvement: a functional magnetic resonance imaging study. Biol Psychiatry 2005;57:901-10.

33 Schwartz JM, Stoessel PW, Baxter LR, et al. Systematic changes in cerebral glucose metabolic rate after successful behavior modification treatment of obsessivecompulsive disorder. Arch Gen Psychiatry 1996;53:109-13.

34 Yamanishi T, Nakaaki S, Omori IM, et al. Changes after behavior therapy among responsive and nonresponsive patients with obsessive-compulsive disorder. Psychiatry Res 2009;172:242-50.

35 Nakatani E, Nakgawa A, Ohara Y, et al. Effects of behavior therapy on regional cerebral blood flow in obsessive-compulsive disorder. Psychiatry Res 2003:124:113-20.

36 Whiteside SPH, Abramowitz JS, Port JD. The effect of behavior therapy on caudate $\mathrm{N}$-acetyl-L-aspartic acid in adults with obsessive-compulsive disorder. Psychiatry Res 2012;201:10-16

37 Whiteside SPH, Abramowitz JS, Port JD. Decreased caudate N-acetyl-L-aspartic acid in pediatric obsessive-compulsive disorder and the effects of behavior therapy. Psychiatry Res 2012;202:53-9.

38 Benazon NR, Moore GJ, Rosenberg DR. Neurochemical analyses in pediatric obsessive-compulsive disorder in patients treated with cognitive-behavioral therapy. J Am Acad Child Adolesc Psychiatry 2003:42:1279-85.

39 Huyser C, van den Heuvel OA, Wolters LH, et al. Increased orbital frontal gray matter volume after cognitive behavioural therapy in paediatric obsessive compulsive disorder. World J Biol Psychiatry 2013;14:319-31.

40 Rosenberg DR, Benazon NR, Gilbert A, et al. Thalamic volume in pediatric obsessivecompulsive disorder patients before and after cognitive behavioral therapy. Biol Psychiatry 2000;48:294-300.

41 Cyr M, Pagliaccio D, Yanes-Lukin P, et al. Altered network connectivity predicts response to cognitive-behavioral therapy in pediatric obsessive-compulsive disorder. Neuropsychopharmacology 2020;45:1232-40.

42 Atmaca M, Yildirim H, Yilmaz S, et al. Orbito-Frontal cortex and thalamus volumes in the patients with obsessive-compulsive disorder before and after cognitive behavioral therapy. Int J Psychiatry Med 2018;53:243-55.

43 Zhong Z, Yang X, Cao R, et al. Abnormalities of white matter microstructure in unmedicated patients with obsessive-compulsive disorder: changes after cognitive behavioral therapy. Brain Behav 2019;9:e01201.

44 Li P, Yang X, Greenshaw AJ, et al. The effects of cognitive behavioral therapy on resting-state functional brain network in drug-naive patients with obsessivecompulsive disorder. Brain Behav 2018;8:e00963.

45 Morgiève M, N'Diaye K, Haynes WIA, et al. Dynamics of psychotherapy-related cerebral haemodynamic changes in obsessive compulsive disorder using a personalized exposure task in functional magnetic resonance imaging. Psychol Med 2014:44:1461-73.

46 Suetens K, Nuttin B, Gabriëls L, et al. Differences in metabolic network modulation between capsulotomy and deep-brain stimulation for refractory obsessive-compulsive disorder. J Nucl Med 2014:55:951-9.

47 Zuo C, Ma Y, Sun B, et al. Metabolic imaging of bilateral anterior capsulotomy in refractory obsessive compulsive disorder: an FDG PET study. J Cereb Blood Flow Metab 2013;33:880-7. 
48 Cecconi JP, Lopes AC, Duran FLdeS, et al. Gamma ventral capsulotomy for treatment of resistant obsessive-compulsive disorder: a structural MRI pilot prospective study. Neurosci Lett 2008;447:138-42.

49 Rauch SL, Kim H, Makris N, et al. Volume reduction in the caudate nucleus following stereotactic placement of lesions in the anterior cingulate cortex in humans: a morphometric magnetic resonance imaging study. J Neurosurg 2000;93:1019-25.

50 Kim MC, Lee TK, Son BC, et al. Regional cerebral blood flow changes in patients with intractable obsessive compulsive disorders treated by limbic leukotomy. Stereotact Funct Neurosurg 2001;76:249-55.

51 Nuttin B, Wu H, Mayberg $\mathrm{H}$, et al. Consensus on guidelines for stereotactic neurosurgery for psychiatric disorders. J Neurol Neurosurg Psychiatry 2014;85:1003-8.

52 Attiullah N, Eisen JL, Rasmussen SA. Clinical features of obsessive-compulsive disorder. Psychiatr Clin North Am 2000;23:469-91.

53 Hurwitz TA, Honey CR, McLeod KR, et al. Hypoactivity in the Paraterminal gyrus following bilateral anterior capsulotomy. Can J Psychiatry 2020;65:46-55.
54 Yin D, Zhang C, Lv Q, et al. Dissociable Frontostriatal connectivity: mechanism and predictor of the clinical efficacy of capsulotomy in obsessive-compulsive disorder. Biol Psychiatry 2018;84:926-36.

55 Le Jeune F, Vérin M, N'Diaye K, et al. Decrease of prefrontal metabolism after subthalamic stimulation in obsessive-compulsive disorder: a positron emission tomography study. Biol Psychiatry 2010;68:1016-22.

56 Abelson JL, Curtis GC, Sagher 0, et al. Deep brain stimulation for refractory obsessivecompulsive disorder. Biol Psychiatry 2005;57:510-6.

57 Van Laere K, Nuttin B, Gabriels L, et al. Metabolic imaging of anterior capsular stimulation in refractory obsessive-compulsive disorder: a key role for the subgenual anterior cingulate and ventral striatum. J Nucl Med 2006;47:740-7.

58 Rauch SL, Dougherty DD, Malone D, et al. A functional neuroimaging investigation of deep brain stimulation in patients with obsessive-compulsive disorder. J Neurosurg 2006;104:558-65.

59 Figee M, Luigjes J, Smolders R, et al. Deep brain stimulation restores frontostriatal network activity in obsessive-compulsive disorder. Nat Neurosci 2013;16:386-7. 